LaQuita M. Jones, DO, Christopher Regan, DO, Kimberly Wolf, DO, Jenifer Bryant, DO, Alexander Rakowsky, MD, Melissa Pe, DO and Dane A. Snyder*, MD

\title{
Effect of osteopathic manipulative treatment on pulmonary function testing in children with asthma
}

https://doi.org/10.1515/jom-2020-0040

Received April 15, 2020; accepted March 9, 2021;

published online May 7, 2021

\section{Abstract}

Context: Asthma is a leading cause of pediatric chronic illness, and poor disease control can lead to decreased quality of life and impaired academic performance. Although osteopathic manipulative treatment (OMT) has been shown to have positive effects on pulmonary function in adult patient populations, less is known about its impact in children.

Objectives: To evaluate changes in pulmonary function testing (PFT) in pediatric patients on the same day they received OMT compared with $\mathrm{PFT}$ in those who received usual care.

Methods: We recruited patients between the ages of 7-18 years with a diagnosis of asthma who were receiving routine care at a primary care asthma clinic and had undergone baseline spirometry. Patients were excluded if they met any of the following criteria: clinical indication for pre- and postbronchodilator spirometry on the day of their visit, albuterol use in the last 8 hours, oral steroid use in the previous 2 weeks, or diagnosis of asthma exacerbation in the previous 4 weeks. Eligible patients were then

*Corresponding author: Dane A. Snyder, MD, Department of Pediatrics, Nationwide Children's Hospital, Ohio State University, 700 Children's Drive, Columbus, OH 43205-2664, USA,

E-mail: dane.snyder@nationwidechildrens.org

LaQuita M. Jones, DO, Cincinnati Children's Hospital, Cincinnati, $\mathrm{OH}$, USA

Christopher Regan, DO and Alexander Rakowsky, MD, Department of Pediatrics, Nationwide Children's Hospital, Ohio State University, Columbus, $\mathrm{OH}$, USA

Kimberly Wolf, DO, Touro University California College of Osteopathic Medicine, Vallejo, CA, USA

Jenifer Bryant, DO, Pediatric Associates, Riverview, FL, USA

Melissa Pe, DO, Christiana Care Pediatric Associates, Newark, DE, USA randomized to either an OMT or a control group. Patients in the OMT group were treated with rib raising and suboccipital release in addition to standard asthma care, while control group patients received standard care only. A second PFT was performed for patients in both groups at the end of the visit. OMT was performed by multiple osteopathic pediatric residents specifically trained for this study. Change in spirometry results (forced vital capacity [FVC], forced expiration volume in 1 second [FEV1], FVC/FEV1, and forced expiratory flow 25-75\%) were then compared. Results: The study population included 58 patients: 31 (53.4\%) were assigned to the OMT group and 27 (46.6\%) were assigned to the standard of care group. Patients who received OMT had greater improvement in all spirometry values compared to the usual group; however, these changes were not statistically significant.

Conclusions: The benefits of OMT on short term spirometry results in pediatric asthma patients remain unclear.

Keywords: asthma; chronic illness; OMT; osteopathic manipulative treatment; pediatrics; rib raising; suboccipital release.

More than 6.5 million children in the United States have a current diagnosis of asthma [1], and during 2017, more than half of all pediatric patients with asthma experienced at least one asthma attack in the preceding 12 months [1]. Poor asthma control leads to a greater risk of hospitalization and emergency department (ED) utilization among pediatric patients. Moreover, poor asthma control has been shown to increase school absence and impair school performance [2]. According to the American Thoracic Society, national costs per asthma patient exceed $\$ 3,200$ annually in medical expenses, missed school and work days, and early deaths [2]. Optimizing asthma control has been a key initiative at Nationwide Children's Hospital ( $\mathrm{NCH}$ ), a large, freestanding pediatric tertiary care center located in Columbus, Ohio.

Osteopathic manipulative treatment (OMT) is a noninvasive, low cost therapeutic option used to treat a 
variety of medical ailments, including asthma. A great deal of anecdotal evidence exists to support the use of OMT in patients with asthma; however, there is very little published, evidence based data to support its efficacy in children. Furthermore, there is a paucity of data showing the impact of OMT on pulmonary function tests (PFTs) [3], the gold standard for objective measurement of pulmonary function and asthma control. PFTs are commonly used to assess asthma control, classify asthma severity, and drive decisions about asthma management. Two reviews concluded that insufficient evidence was available to demonstrate that manual therapy could benefit patients with asthma, and that further adequately sized, randomized, controlled trials were needed [4, 5]. Therefore, evaluating the effect of OMT on pediatric asthma care is of interest to $\mathrm{NCH}$.

The current study builds upon the authors' preliminary work [3] examining effects of suboccipital release and rib raising. In that patient cohort $(\mathrm{n}=8)$, the OMT group experienced improvements in forced expiratory flow $25-75 \%$ (FEF 25-75\%) and forced expiration volume in 1 second (FEV1), and did not report adverse events, though statistical significance was not reached [3]. Given these results, the current study was developed to evaluate the effects of OMT in a larger patient cohort.

The objective of our study was to determine whether OMT would improve same day repeat PFT results to a greater extent than standard therapy alone. We compared FEV1, forced vital capacity (FVC), FEV1/FVC, and FEF $25-75 \%$ changes from baseline PFT to a second PFT conducted later in the same day in both groups.

\section{Methods}

This study was approved by the NCH Institutional Review Board (IRB 14-00790) and registered as a clinical trial (ClinicalTrials.gov, NCT04764877).

Pediatric patients with asthma who were seen in the NCH primary care asthma specialty clinic and completed a PFT during the visit were recruited and randomized into two groups: OMT intervention (in addition to usual care) and usual care only. Subjects were recruited between February 2015 and December 2016. Patients between 7 and 18 years of age with a diagnosis of asthma, scheduled routine asthma care at the office's asthma specialty clinic, and at least one PFT prior to participation were eligible for inclusion. Patients were excluded if they met any of the following criteria: clinical indication for pre- and postbronchodilator spirometry on day of visit, albuterol use in the previous 8 hours, oral steroid use in the previous 2 weeks, or a diagnosis of asthma exacerbation in the previous 4 weeks.

Randomization was completed by the study coordinator at study onset, independent of the authors, and was completed utilizing an automated simple randomization tool that assigned each patient a number for either the OMT group ("1") or the usual care group ("2"). The order of randomization, by subject number, was determined at that time for the entire study. After screening patients for inclusion, the study coordinator obtained consent. Written informed consent was obtained from the patients' parents and assent was also obtained from patients 9 years and older. If consent was obtained, the study coordinator assigned the patient to the next subject number in sequential order (determining OMT or usual care group based on prior randomization protocol) and notified the physician team, who then provided standard care with or without OMT. Patients in both groups continued to receive standard care routinely provided during an asthma specialty clinic visit, including standardized clinician assessment and documentation, education provided by a multidisciplinary team, and tablet education tutorials. All participants from both groups underwent a second PFT at the end of the clinic visit. As a routine part of the visit, patients received an explanation of baseline PFT results, discussion of medications, spacer teaching, basic asthma education, and home instructions including an individualized asthma action plan. Spirometry was performed by a respiratory therapist trained in pediatric care, who was blinded to patient study group assignment. All subjects completed the study.

Patients randomized to the OMT group received the same care as the control group, with the addition of a standard OMT protocol performed by a resident doctor of osteopathic medicine (D.O.). The resident performing the treatment was one of 12 osteopathic pediatric residents in NCH's pediatric residency program, all of whom participated in the study. In order to standardize the treatment technique as much as possible, all participating residents were trained by a single attending physician (K.W.) specializing in pediatric OMT. Two techniques were used in each intervention patient: rib raising and suboccipital release. Rib raising was performed in the seated position with the physician treating the rib cage bilaterally. For standardization, residents were trained to complete two full cycles, moving caudad and cephalad along the rib angles. Suboccipital release was performed for 45 seconds on a supine patient with the physician's finger pads contacting the suboccipital musculature. These treatments were performed without a specific assessment for somatic dysfunction to standardize treatment across multiple providers, and to evaluate potential benefit regardless of physical exam findings. The application of these techniques was performed to alter autonomic tone rather than treating for specific somatic dysfunction or tenderness, asymmetry, restricted motion, or tissue texture changes. For this reason, the standardized application for suboccipital release was based on time rather than tissue response. Likewise, the rib raising technique was based on completion of two passes over the entire rib cage rather than specific assessment for rib motion or tissue changes in the region.

Results from baseline and second PFTs were collected. Four distinct PFT measures were analyzed: FEF $25-75 \%$, FVC, FEV1, and FEV1/FVC. Other patient characteristics collected as possible control variables were age, sex, asthma classification (intermittent, mild persistent, moderate persistent, severe persistent), race (self reported at the patient's first appointment), presence of comorbidities (obesity, attention deficit hyperactive disorder [ADHD], and other behavior problems), and asthma control test (ACT) score at baseline. The ACT form was completed by both parents and patients. For patients aged 4 to 11 years, the ACT in divided into questions for both parents and patients. The ACT is intended to be completed by only the patient if they are 12 years or older; however, there is no way to know for certain if a parent assists or completes the form for the patient, as it is completed prior to the physician entering the room. 
Descriptive statistics (frequencies/percentages for categorical variables; means/standard deviations [SD] for continuous variables) were performed for demographic and baseline clinical characteristics. Two sample t-tests were used to compare baseline means between the groups for continuous variables, and chi square tests were used to compare baseline frequencies for categorical variables. A p-value $<0.05$ was considered statistically significant. No significant differences were noted in baseline characteristics of the two groups.

In addition, we compared the change from baseline in FEF 25-75\%, FVC, FEV1 and FEV1/FVC, utilizing analysis of covariance to assess the differences between patients who received both OMT and usual care vs. those who received only usual care. We also stratified data based upon the presence or absence of persistent asthma, which was determined by the encounter diagnosis on the day of the study visit and recorded on the data sheet at that time. During this analysis, we did not further control for baseline characteristics since all baseline data was similar between the two groups. Statistics were completed by an NCH biostatistician assigned to this project who was blinded to the respective groups.

\section{Results}

The study population included 58 patients: 31 (53.4\%) in the OMT group and $27(46.6 \%)$ in the control group. Overall, $31(53.4 \%)$ patients were male and 27 (46.6\%) were female. The patients' mean age was 10.8 years old (range, 7-17 years) and $27(46.6 \%)$ also had at one of the following comorbidities: obesity, ADHD, or any other diagnosed behavioral problem in the patient's history. At the time of enrollment, $22(37.9 \%)$ patients were classified as having intermittent asthma, while $36(62.1 \%)$ were classified as having persistent asthma. When comparing demographics and baseline clinical characteristics between the two groups, no significant differences were observed (Table 1).

\section{PFT at baseline}

For three of the four measures (FEF 25-75\%, FVC, and FEV1), baseline PFT mean values were higher for the OMT group than the control group. However, these differences were not statistically significant ( $>0.05$ for all PFT measures at baseline; Table 2).

\section{Change in PFT between baseline and second measurement}

Patients who received OMT ( $\mathrm{n}=31)$ had greater mean increases in FEF 25-75\%, FVC, and FEV1 compared with the 27 patients in the usual care group who did not receive OMT; however, these differences did not reach the level of statistical significance. The difference in these results
Table 1: Demographics for the OMT group vs. control group.

\begin{tabular}{|c|c|c|c|}
\hline Characteristic & $\begin{array}{l}\text { OMT } \\
(n=31) \\
n(\%)\end{array}$ & $\begin{array}{l}\text { Control } \\
(n=27) \\
n(\%)\end{array}$ & p-Value \\
\hline \multicolumn{4}{|l|}{ Sex } \\
\hline Male & $19(61.3 \%)$ & $12(44.4 \%)$ & $0.20^{\mathrm{a}}$ \\
\hline Female & $12(38.7 \%)$ & $15(55.6 \%)$ & \\
\hline Age, years (range) & $10.9(7-18)$ & $10.6(7-17)$ & 0.69 \\
\hline \multicolumn{4}{|l|}{ Race } \\
\hline Biracial & $1(3.2 \%)$ & $4(14.8 \%)$ & $0.12^{\mathrm{a}}$ \\
\hline Black & $13(41.9 \%)$ & $6(22.2 \%)$ & $0.11^{\mathrm{a}}$ \\
\hline White & $15(48.4 \%)$ & $14(51.8 \%)$ & $0.79^{\mathrm{a}}$ \\
\hline Hispanic & $2(6.5 \%)$ & $3(11.1 \%)$ & $0.53^{\mathrm{a}}$ \\
\hline \multicolumn{3}{|l|}{ comorbidities ${ }^{\mathrm{b}}$ present } & $0.95^{\mathrm{a}}$ \\
\hline Obesity only & $5(16.1 \%)$ & $6(22.2 \%)$ & $0.55^{\mathrm{a}}$ \\
\hline $\begin{array}{l}\text { ADHD or behavioral } \\
\text { problem only }\end{array}$ & $6(19.4 \%)$ & $4(14.8 \%)$ & $0.65^{\mathrm{a}}$ \\
\hline $\begin{array}{l}\text { Both obesity and ADHD/ } \\
\text { behavioral problem }\end{array}$ & $3(9.7 \%)$ & $3(11.1 \%)$ & $0.86^{\mathrm{a}}$ \\
\hline $\begin{array}{l}\text { Subjects with no } \\
\text { comorbidities }\end{array}$ & $17(54.8 \%)$ & $14(51.9 \%)$ & $0.82^{\mathrm{a}}$ \\
\hline Intermittent asthma & $13(42.0 \%)$ & $9(33.0 \%)$ & $0.50^{\mathrm{a}}$ \\
\hline $\mathrm{ACT}$ at baseline, mean (SD) & $20.3(4.4)$ & $21.8(2.8)$ & 0.12 \\
\hline
\end{tabular}

ap-value reported for categorical data; bobesity or ADHD/behavioral problem. ACT, asthma control test; $A D H D$, attention deficit hyperactive disorder; OMT, osteopathic manipulative treatment; SD, standard deviation.

Table 2: Baseline PFT results for OMT group vs. control group.

\begin{tabular}{lrrrrrr}
\hline Baseline PFT & \multicolumn{2}{c}{ OMT $(\mathbf{n = 3 1 )}$} & & \multicolumn{2}{c}{ Control $(\mathbf{n = 2 7 )}$} & p-Value $^{\text {a }}$ \\
\cline { 2 - 3 } & Mean & SD & & Mean & SD & \\
\hline FEF 25-75\% & 91.5 & 28.2 & & 88.4 & 26.3 & 0.67 \\
FVC & 104.9 & 11.5 & & 99.1 & 12.1 & 0.07 \\
FEV1 & 100.7 & 14.2 & & 95.3 & 13.1 & 0.14 \\
FEV1/FVC & 83.6 & 7.9 & & 85.4 & 10.9 & 0.47 \\
\hline
\end{tabular}

${ }^{\mathrm{a}} \mathrm{p}$-Value based on 2 sample t-test. FEF $25-75 \%$, forced expiratory flow $25-75 \%$; FEV1, forced expiration volume in 1 second; FVC, forced vital capacity; OMT, osteopathic manipulative treatment; PFT, pulmonary function test; SD, standard deviation.

remained consistent among the 36 patients with persistent asthma and the 22 patients with intermittent asthma, based on diagnosis at the time of their study visit.

For patients in the OMT group, the mean FEF 25-75\% increased $4.4 \%(91.5-95.5 \%$ predicted) vs. a $1.2 \%$ mean decrease $(88.4-87.3 \%)$ for patients in the control group $(\mathrm{p}=0.05$; Table 3$)$. Within the OMT group, a $4.2 \%$ increase (106.7-111.2) in mean FEF 25-75\% was observed in the 13 patients with intermittent asthma, compared with a $1.6 \%$ (100.7-99.1) mean decrease in nine patients with 
Table 3: PFT results for OMT group vs. control group.

\begin{tabular}{|c|c|c|c|c|c|c|c|c|c|}
\hline \multirow[t]{3}{*}{ Outcomes measure } & \multicolumn{4}{|c|}{ OMT $(n=31)$} & \multicolumn{4}{|c|}{ Control $(n=27)$} & \multirow[t]{3}{*}{ p-Value ${ }^{a}$} \\
\hline & \multicolumn{2}{|c|}{ Baseline PFT } & \multicolumn{2}{|c|}{ Second PFT } & \multicolumn{2}{|c|}{ Baseline PFT } & \multicolumn{2}{|c|}{ Second PFT } & \\
\hline & Mean & SD & Mean & SD & Mean & SD & Mean & SD & \\
\hline FEF $25-75 \%$ & 91.5 & 28.2 & 95.5 & 28.6 & 88.4 & 26.3 & 87.3 & 26.4 & 0.05 \\
\hline FVC & 104.9 & 11.5 & 107.4 & 12.4 & 99.1 & 12.1 & 100.2 & 12.6 & 0.26 \\
\hline FEV1 & 100.7 & 14.2 & 103.1 & 14.1 & 95.3 & 13.1 & 95.6 & 13.7 & 0.06 \\
\hline FEV1/FVC & 83.6 & 7.9 & 83.6 & 8.1 & 85.4 & 10.9 & 84.7 & 10 & 0.51 \\
\hline
\end{tabular}

${ }^{a}$ p-Value based on analysis of covariance (ANCOVA). FEF 25-75\%, forced expiratory flow 25-75\%; FEV1, forced expiration volume in 1 second; FVC, forced vital capacity; OMT, osteopathic manipulative treatment; PFT, pulmonary function test; SD, standard deviation.

intermittent asthma in the control group $(\mathrm{p}=0.216$; Figure 1). The 18 patients in the OMT group with persistent asthma had a mean increase in FEF $25-75 \%$ of $4.6 \%$ (80.584.2) after OMT; the 18 patients in the control group with persistent asthma had a mean decrease in FEF $25-75 \%$ of $1.1 \%$ (82.3-84.1) ( $\mathrm{p}=0.184$; Figure 1).

FVC increased by a mean of $2.4 \%$ (104.9\% predicted to $107.4 \%$ ) in all patients who received OMT, compared with a mean increase of 1.1\% (99.1-100.2\%) in the control group $(p=0.26)$. Patients in the OMT group with intermittent and persistent asthma had mean FVC increases of 2.2\% (102.5-104.8) and 2.5\% (106.7-109.3), respectively. In the control group, patients with intermittent asthma had a mean decrease of $0.3 \%$ (98.3-98.0; $\mathrm{p}=0.147)$ while patients with persistent asthma had an average increase of $1.8 \%$ (99.5-101.3; $\mathrm{p}=0.683$ ).

Mean FEV1 increased 2.4\% (100.7\% predicted to $103.1 \%$ ) in all patients who received OMT vs. a mean increase of $0.3 \%(95.3-95.6 \%)$ in patients from the control group ( $\mathrm{p}=0.06)$. Patients in the OMT group with intermittent and persistent asthma had a mean increase in FEV1 of 1.9\% (104.3-106.3) and 2.8\% (98.1-100.8), respectively, while patients in the control group with intermittent and persistent asthma had a mean decrease 1.4\% (100.3-98.9; $\mathrm{p}=0.068)$ and a mean increase of $1.3 \%(92.8-94.0 ; \mathrm{p}=0.33)$, respectively.

We observed minimal change in both groups in FEV1/FVC. Overall, OMT group patients had no mean change (83.6-83.6) and usual care group patients had a mean decrease of $0.8 \%(85.4-84.7)$ in this metric $(\mathrm{p}=0.51)$. We also observed a $0.1 \%$ mean increase $(87.7-87.8)$ in OMT group patients with intermittent asthma and a $0.2 \%$ mean decrease (80.7-80.5) in patients with persistent asthma within the OMT group. Usual care group patients with intermittent asthma had a $1.1 \%$ mean decrease (88.9-87.9) in this ratio $(\mathrm{p}=0.192)$, while patients with persistent asthma receiving usual care had a mean $0.6 \%$ decrease (83.6-83.1; $\mathrm{p}=0.746)$.

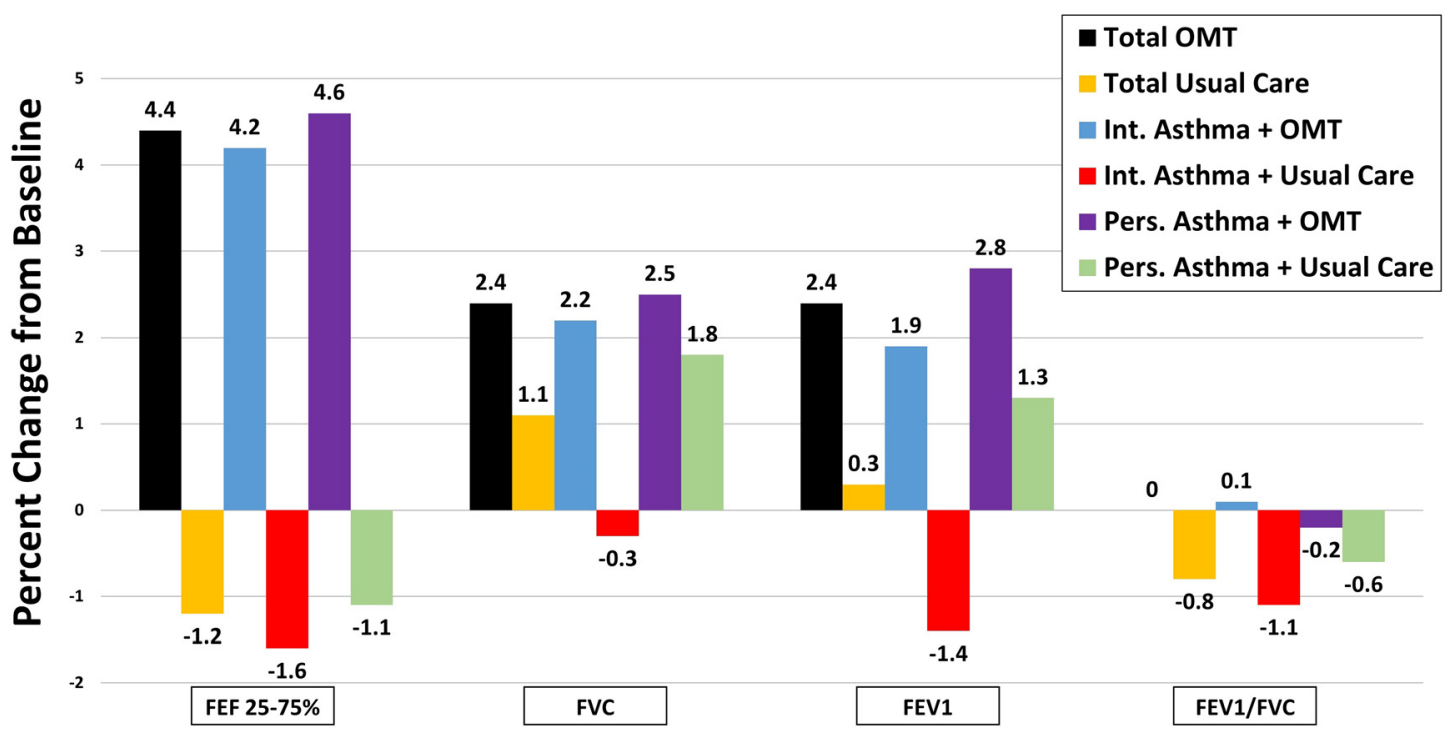

Figure 1: Percent change from baseline pulmonary function test, osteopathic manipulative treatment (OMT) group vs. control group. 


\section{Discussion}

This study describes a trial to compare the change in same day PFT results between pediatric patients who received OMT in addition to usual asthma care vs. those in a control group, who received usual care only. Although we observed a greater improvement in spirometry values in patients who received OMT, these results did not reach the level of statistical or clinical significance. In addition, we found no statistical difference in repeat PFT performance between patients in both groups with intermittent or persistent asthma.

Other studies have shown significant improvements in spirometry in adult patients after OMT, and the importance of spirometry to accurately assess asthma control. Lima et al. [6] demonstrated a significant improvement in spirometry results in 100 healthy subjects after a single manipulative treatment session, and Bye et al. [7] demonstrated in a study of 65 patients that those lacking spirometry assessment had an overestimation of their asthma control and an underestimation of their level of obstructive disease.

Specific to asthma, Guiney et al. [8] demonstrated improvements in peak flow meter results after OMT in 140 asthmatic patients, but did not assess impact on PFTs. In 2013, we conducted a pilot study that showed a correlation between OMT use in patients with asthma and improvement in PFTs [3]. However, that study [3] lacked statistical significance due to small sample size and it did not factor in a number of variables, including potential medication adjustments or severity of disease. The objective of the present study was to expand on the earlier pilot with a randomized controlled study to evaluate the effect of OMT on PFTs. While other spirometry specific pediatric studies using OMT as an intervention have not been reported, many studies have shown improvements in other lung diseases (such as pneumonia and cystic fibrosis) with the use of manual therapy [8-11]. Furthermore, other studies have shown that manual therapy as an adjunct to treatment can improve spirometry results in patients with obstructive lung diseases other than asthma, such as chronic obstructive pulmonary disease [12, 13]. Moreover, others have shown improvement in spirometry results in healthy subjects following manual therapy treatment [14]. Therefore, we hypothesized that OMT would show a similar benefit for patients with asthma, demonstrated by improved pulmonary function results. The National Heart, Lung, and Blood Institute now recommends spirometry evaluation for all patients with asthma every 1 to 2 years, and current guidelines include spirometry results as a way to properly classify patients with asthma [15]. Thus, given spirometry's role as an important marker of asthma control, demonstrating improvements utilizing OMT in pediatric populations could lead to enhanced disease management options.

We chose specific PFT measures - FVC, FEV1, FEV1/FVC, and FEF 25-75\% - given their importance in evaluating asthma control. FVC is an important indicator of overall lung capacity, and can indicate restrictive breathing if abnormal [16]. FEV1 is known to correlate with severity of airways obstruction and is a useful measure of a patient's current asthma control [16]. The ratio of FEV1 to FVC is also used to determine the type of lung disease present, while the FEF $25-75 \%$ is a good indicator of small airway function [16]. Normal values for FVC, FEV1 and FEV1/FVC are $>80 \%$ of the predicted value based on the patient's age, weight, and height [15]. An FEF 25-75\% of $>60 \%$ predicted value is considered normal in children [15]. To determine whether an intervention produces a significant change, three parameters - FVC, FEV1 and FEV1/FVC - must increase by $12 \%$ or more, while FEF $25-75 \%$ must show an increase of greater than $20 \%$ $[15,17]$. While we did not show such significant changes in the pre- and post OMT PFTs, this may be due to the stable nature of the disease for the patients enrolled in our study.

The specific OMT techniques were chosen with consideration of the five models of osteopathic medicine. The biomechanical model focuses on the structural aspects of the patient that may be playing a role in their complaint. For this study, rib raising eases rib restrictions by articulating the rib heads with an anterolateral motion, which can contribute to increased rib mobility. This improved mobility allows for more efficient and effective respiratory effort with increased rib cage excursion. Given that the six most inferior ribs also have direct diaphragmatic attachments, this technique also influences the biomechanics of the diaphragm to further improve respiratory effort.

In this study, the techniques of suboccipital release and rib raising were chosen with a goal of normalizing autonomic tone. The sympathetic chain ganglia are influenced through rib raising via fascial attachments and close physical proximity to the rib heads. The sympathetic innervation to the lungs originates at the levels of $\mathrm{T} 1$ down to T5/6. The sympathetic influence on the lungs typically causes bronchodilation and vasoconstriction. The parasympathetic innervation to the lungs is supplied by the vagus nerve, which courses through the suboccipital region after exiting the skull through the jugular foramen and is addressed with the suboccipital release technique. Increased parasympathetic tone allows for vasodilation 
and bronchoconstriction, and improved parasympathetic flow also thins secretions which can obstruct inflamed airways. The weight of the patient's head helps the musculature in this region to relax and ultimately frees the path of the vagus nerve. Somatic dysfunction in either the upper thoracic spine or suboccipital regions will increase afferent input and allow for facilitation and development of viscerosomatic reflexes that can worsen an asthma patient's clinical condition. Both study techniques have been shown to influence autonomic tone [18-22].

In the respiratory-circulatory model, the physician aims to maximize the cardiovascular and respiratory function of the patient. Rib raising, as discussed above, through its influence on the rib cage and diaphragm allows for improved respiratory effort. Rib raising may also encourage expectoration and aid in dislodging the thick mucus present in many asthmatic patients' airways. The diaphragm is critical to the efficiency of respiration and serves as a vital component of the circulatory system through its pumping action. This also allows for better immunologic and lymphatic function, which ties into the metabolic model. This model focuses on decreasing energy demands of the body, so rib raising assists in this model by improving efficiency of respiration.

The behavioral model - although not directly addressed with the OMT performed in this study - was addressed in the asthma clinic setting by the physician managing the patients' asthma. In addition to medication management, this clinic focused on ways to reduce asthma exacerbations, ED visits, and hospitalizations with a variety of behavioral interventions. These include extensive asthma education, the development of individualized asthma action plans, and even preparing "back to school" kits for each child to take to school (including their action plan and an extra inhaler).

The two simple and brief techniques performed in this study allowed for a balancing of the patient's autonomic nervous system, decreased afferent input into the viscerosomatic reflex arc, and improved biomechanics to ultimately improve the patient's respiratory status, both acutely and long term.

\section{Limitations}

In clinics that routinely practice OMT, it may take multiple treatments to reduce or eliminate the viscerosomatic effects of any dysfunction; however, response has been demonstrated with as little as one application [17-21]. Statistically significant improvement may have been observed if this study were conducted over multiple visits with multiple treatments. Another limitation of our study is the use of two nonspecific treatments. In true practice, OMT is more individualized and often includes more techniques to assess and respond to somatic dysfunction. This was not assessed in this trial, as we attempted to standardize protocol among multiple providers, and tissue response was not evaluated, providing an additional limitation. Future studies could include a focused osteopathic examination pre- and post OMT intervention to document the presence and severity of somatic dysfunction and tissue response with the application of OMT. Similar studies investigating the effects of OMT on the respiratory system have included a broader range of treatments, including muscle energy, myofascial release, balanced ligamentous tension, and lymphatic pumps [8-12]. Both Guiney et al. [8] and Zanotti et al. [12] showed improvement in objective peak flow measurements using a variety of osteopathic manipulative treatments that were individualized for each patient. However, this approach could introduce more variation in examiner findings, types of techniques used, and potential response to treatment. We limited intervention to these two techniques to reduce variation and standardize treatment given the number of resident providers who would be administering the treatment.

There were several limitations to our study which could be improved upon in future work. First, while much larger than our 2013 pilot study $(n=8)$, [3] our sample size was still quite small with 58 subjects. The study was underpowered and this may have contributed to the lack of statistical significance of the improvement in PFTs in the OMT group. A power calculation performed after the study concluded showed that, in order to detect statistical significance in FEV1 (assuming data remained consistent in the two groups), 74 subjects would need to be enrolled. FEV1 was chosen for power calculation given its importance in assessment of pulmonary function [16]. Several factors contributed to our small sample, including a gap in recruitment efforts due to the departure of first study coordinator, which delayed subject enrollment for more than 9 months while a replacement was hired. Additionally, several other measures of treatment efficacy could have been included if the study period were longer. For instance, this study did not investigate long term measures of improvement, such as improvement in ACT scores at subsequent visits, reduction in ED or urgent care visits for acute exacerbations, or subjective improvement in symptoms and quality of life. These are important factors when considering asthma therapy, as they contribute to medical decision making and patient satisfaction, and ultimately patient adherence. While not formally surveyed, the patients did not report any adverse events after OMT 
related to this study. Adding longer term follow up by waiting at least 24 hours prior to repeat spirometry could have changed the outcomes (e.g., greater improvement in PFT or quality of life). There is inherent logistical difficulty with including long term measures, such as residents' limited time in clinics and inability to conduct follow up phone surveys due to other clinical demands. The logistic challenges of resident schedules and frequent transition to other organizations after residency are also reflected in the delay between the study period and manuscript submission. Furthermore, there may be challenges with patient adherence, specifically with follow up visits to repeat PFTs or collection of ACT forms.

The study was initially designed to include onemonth follow up telephone call to assess asthma control. Given logistical challenges such as resident schedules and the duration of project enrollment, this was not completed.

To enhance recruiting success, future studies should consider utilizing a setting that involves attending physicians who practice full time in the office or one with more administrative research resources to complete these tasks. We were also limited by the infrequency (once per week) of the asthma specialty clinic. Future studies would likely benefit from the ability to recruit on a more consistent (perhaps daily) basis or by designing a multicenter trial.

Lastly, the lack of blinding to patient and medical provider limited the study further. Considering alternative methods to improve blinding of patients may be beneficial to future studies.

The techniques used in this study were quickly taught and reproducible by multiple osteopathic providers. The study itself was easily incorporated in a busy clinical practice with multiple providers. The techniques took less than 3 minutes to complete, minimizing any disruption in regular patient flow, and are reproducible for future studies.

\section{Conclusions}

Patients who received OMT in addition to usual care had moderate increases in same day PFT metrics vs. patients who only received usual care; however, the differences were neither clinically nor statistically significant and could have been the result of normal variation. Further investigation in a larger cohort is necessary to recommend broad scale application of these techniques in clinical practice.
Research funding: The current study was supported by the Ohio University Heritage College of Osteopathic Medicine CORE seed funding.

Author contributions: All authors provided substantial contributions to conception and design, acquisition of data, or analysis and interpretation of data; Drs. Jones and Snyder drafted the article or revised it critically for important intellectual content; all authors gave final approval of the version of the article to be published; and all authors agree to be accountable for all aspects of the work in ensuring that questions related to the accuracy or integrity of any part of the work are appropriately investigated and resolved.

Competing interests: None reported.

Informed consent: Written informed consent was obtained from the patients' parents and assent was also obtained from patients 9 years of age and older.

Ethical approval: This study was approved by the Nationwide Children's Hospital Institutional Review Board (IRB 14-00790) and registered as a clinical trial (ClinicalTrials.gov, NCT04764877).

\section{References}

1. Centers for Disease Control and Prevention. Asthma. Available from: cdc.gov/ [Accessed 30 Oct 2020].

2. Nurmagambetov T, Kuwahara R, Garbe P. The economic burden of asthma in the United States, 2008-2013. Ann Am Thorac Soc 2018;15:348-56.

3. Pe M, Jones L, Snyder D, Jenkins J. The effect of OMT on PFT results in children with asthma. ACOPeds Abstr 2013.

4. Posadzki P, Lee MS, Ernst E. Osteopathic manipulative treatment for pediatric conditions: a systematic review. Pediatrics 2013; 132:140-52.

5. Hondras MA, Linde K, Jones AP. Manual therapy for asthma. Cochrane Database Syst Rev 2005:CD001002.

6. Lima IS, Filho O, Cunha F, Ribiero J, Valenti V, de Abreu L. Chest and neck mobilization effects on spirometric responses in healthy subjects. J Manip Physiol Ther 2011;34:622-6.

7. Bye MR, Kerstein $D$, Barsh $E$. The importance of spirometry in the assessment of childhood asthma. Am J Dis Child 1992;146: 977-8.

8. Guiney PA, Chou R, Vianna A, Lovenheim J. Effects of osteopathic manipulative treatment on pediatric patients with asthma: a randomized controlled trial. J Am Osteopath Assoc 2005;105: 7-12.

9. Swender DA, Thompson G, Schneider K, McCoy K, Patel, A. Osteopathic manipulative treatment for inpatients with pulmonary exacerbations of cystic fibrosis: effects on spirometry findings and patient assessments of breathing, anxiety, and pain. J Am Osteopath Assoc 2014;114:450-8.

10. Hodge $L M$. Osteopathic lymphatic pump techniques to enhance immunity and treat pneumonia. Int J Osteopath Med 2012;15:13-21. 
11. Noll DR, Degenhardt BF, Morley TF, Blais FX, Hortos KA, Hensel K, et al. Efficacy of osteopathic manipulation as an adjunctive treatment for hospitalized patients with pneumonia: a randomized controlled trial. Osteopath Med Prim Care 2010;4:2.

12. Zanotti E, Berardinelli P, Bizzarri C, Civardi A, Manstretta A, Rossetti S, et al. Osteopathic manipulative treatment effectiveness in severe chronic obstructive pulmonary disease: a pilot study. Compl Ther Med 2012;20:16-22.

13. Engel RM, Vemulpad SR, Beath K. Short-term effects of a course of manual therapy and exercise in people with moderate chronic obstructive pulmonary disease: a preliminary clinical trial. J Manip Physiol Ther 2013;36:490-6.

14. Engel RM, Vemulpad S. The effect of combining manual therapy with exercise on the respiratory function of normal individuals: a randomized control trial. J Manip Physiol Ther 2007;30: 509-13.

15. National Asthma Education and Prevention Program. Third expert panel on the diagnosis and management of asthma. Expert panel report 3: guidelines for the diagnosis and management of asthma. https://www.ncbi.nlm.nih.gov/books/NBK7232/pdf/ Bookshelf_NBK7232.pdf [Accessed 16 Apr 2021].
16. Enright PL, Lebowitz MD, Cockroft DW. Physiologic measures: pulmonary function tests. Asthma outcome. Am J Respir Crit Care Med 1994;149:S9-18; discussion S9-20.

17. Barreiro TJ, Perillo I. An approach to interpreting spirometry. Am Fam Phys 69 2004:1107-14.

18. Henderson AT, Fisher JF, Blair J, Shea C, Li TS, Bridges KG. Effects of rib raising on the autonomic nervous system: a pilot study using noninvasive biomarkers. J Am Osteopath Assoc 2010;110: 324-30.

19. Fornari M, Carnevali L, Sgoifo A. Single osteopathic manipulative therapy session dampens acute autonomic and neuroendocrine responses to mental stress in healthy male participants. J Am Osteopath Assoc 2017;117:559-67.

20. Henley CE, Ivins D, Mills M, Wen FK, Benjamin BA. Osteopathic manipulative treatment and its relationship to autonomic nervous system activity as demonstrated by heart rate variability: a repeated measures study. Osteopath Med Prim Care 2008;2:7.

21. Giles PD, Hensel KL, Pacchia CF, Smith ML. Suboccipital decompression enhances heart rate variability indices of cardiac control in healthy subjects. J Alternative Compl Med 2013;19:92-6.

22. Emmet D, Nuño V, Pierce-Talsma S. OMT to address the physiologic effects of stress. J Am Osteopath Assoc 2018;118:e11. 